Methods Among all chlamydia tests performed at a woman's first pregnancy-related visit between June 2008 and July 2010, we estimated chlamydia positivity by age, then further stratified by insurance coverage and geographic region.

Results Of 600,990 pregnant women aged $15-44$ years, $61.9 \%$ had private insurance and $34.1 \%$ had Medicaid coverage; $60.8 \%$ resided in the South region; 43.4\% were aged 15-24 years, $26.7 \%$ 25-29 years, and $19.1 \%$ 30-34 years. Chlamydia positivity significantly decreased by age (15-19 years: $10.3 \%$; $20-24$ years: $5.6 \%$; $25-29$ years: $1.9 \%$; $30-34$ years: $0.9 \%$; and $35-44$ years: $0.6 \%)$. The pattern of decreased age-specific positivity was similar among insurance and region subgroups.

Conclusions Our findings of age-specific positivity, derived from a very large number of tests among pregnant women in the United States, suggest that it is more effective to screen younger pregnant women than older ones. Harmonizing CDC and USPSTF recommendations for pregnant women could be explored by review of age-specific positivity data and estimates of prenatal adverse health outcomes caused by chlamydia (miscarriage, preterm birth, and infant mortality) in order to develop consensus regarding quantitative thresholds of these health outcomes.

\subsection{DO YOU HAVE AN STI? FINDINGS FROM A DEDICATED MEN'S SEXUAL HEALTH CLINIC IN ALEXANDRA TOWNSHIP, SOUTH AFRICA}

doi:10.1136/sextrans-2013-051184.0130

1,2,3 D Lewis, 'C Ricketts, 'A Vezi, 'V Maseko. 'National Institute for Communicable Diseases (NHLS), Sandringham, South Africa; ${ }^{2}$ University of the Witwatersrand, Johannesburg, South Africa; ${ }^{3}$ University of Cape Town, Cape Town, South Africa

Background A walk-in weekly men's sexual health clinical service, provided by a male clinical team, was established in 2006.

Methods We analysed new patient episodes at a dedicated men's sexual health clinic in Alexandra Township, South Africa over 6 years (2007-2012). STI syndromes were treated immediately and all men were offered urine-based molecular screening for Neisseria gonorrhoeae (NG), Chlamydia trachomatis (CT), Trichomonas vaginalis (TV) and Mycoplasma genitalium (MG) infections and serological screening for syphilis. Clinical and laboratory data were analysed using STATA ${ }^{\mathrm{TM}}$ version 10

Results Among the 876 new clinical episodes, the most common presentations were genital warts $(432,49.5 \%)$, male urethritis syndrome (188, 21.6\%) and genital ulceration (82, 9.4\%). The proportion of patients attending for genital wart treatment increased over time. Few men (51, 5.9\%) presented as sexual contacts. The patients' peak age range was 25-29 years; only 40 (4.6\%) men were $<20$ years old. HIV testing history was provided by 871 men: $156 / 430$ (36.3\%) men who knew their serostatus were HIV positive but $441(50.6 \%)$ had never tested. Laboratory testing of 822 urine specimens detected 108 (13.1\%) NG, 100 (12.2\%) CT, 51 $(6.2 \%)$ TV and 68 (8.3\%) MG infections. The syndromic approach alone would not have treated 16 (14.8\%) NG, 57 (57.0\%) CT, 46 $(90.2 \%)$ TV and 49 (72.1\%) MG infections. NG/CT infections were most prevalent among those aged 18-19 years old $(34.5 \%$ and $17.2 \%$, respectively); in contrast, TV/MG infections were most prevalent in the $35-39$ year old age group $(1.7 \%$ and $11.8 \%$, respectively). The prevalence of rapid plasma reagin seroreactivity was $2.2 \%$. Overall condom use was $16.9 \%$ with regular partners and $52.5 \%$ with non-regular partners.

Conclusion Asymptomatic STIs, poor clinic attendance by youth, poor uptake of HIV testing by men and low rates of condom use with partners remain challenges for STI management in South Africa.

\subsection{9 - Programme implementation and scaling up}

\section{1 \\ VOICES OF HIV INFECTED CHILDREN AND THEIR FAMILIES}

doi:10.1136/sextrans-2013-051184.0131

'B Panda, ${ }^{2} \mathrm{~K}$ Shaw. ${ }^{1}$ Chandipur Mother \& Child Welfare Society, Kolkata, India; ${ }^{2}$ Bengal Network of Positives (BNP+), Kolkata, India

Chandipur Mother \& Child Welfare Society, in partnership with Bengal Network of People Living with HIV/AIDS tied up with district networks, to achieve the following objectives:

- Building capacity of the Network of People Living with HIV/ AIDS to track children

- Assessing the profile of these children, their families and the extent to which these children are able to access basic services related to health, nutrition and education.

Sixty trained HIV positive network members collected the information in 2010-11. Information collected from the families have HIV/AIDS infected or exposed children. The study team traced 1,639 children in 995 families, out of whom 857 children were reported as HIV infected and 130 were exposed children. To gather more qualitative information, number of FGDs \& consultations were held.

1. $72 \%$ families fall in BPL category and mostly engaged in unorganised sector

2. $29 \%$ of fathers and $33 \%$ of mothers illiterate

3. $65 \%$ mothers not received any PPTCT services during their last pregnancy

4. $11 \%$ transmission through contaminated blood and blood products

5. $60 \%$ children receive care from a single institution

6. $43 \%$ families have spent an out of pocket expense

7. Very few children who were tracked are below 2 years of age, indicating late detection \& initiation of treatment

8. $65 \%$ of eligible children attend school \& $62 \%$ children, $<$ six years are enrolled in ICDS

9. Parents exhibit an inherent fear of stigma and discrimination

The study reveals that most of these children belong to poor socio-economic condition. It emphasises the need to improve existing services in terms of timely access to appropriate medicine, treatment and nutrition.

It also strongly recommends the need to scale up the coverage of services towards virtual elimination of mother to child transmission of HIV. There is need to change in attitude of service providers and society at large to remove stigma and discrimination.

\subsection{FINANCING FREE AND UNIVERSAL ACCESS TO ANTIRETROVIRAL DRUGS IN THE LONG-RUN: ART COST EVOLUTION IN BRAZIL}

doi:10.1136/sextrans-2013-051184.0132

\section{Meiners-Chabin. Ministry of Health, Brasilia, DF, Brazil}

Background Following the international aid crisis, developing country governments have assumed greater financial responsibility in the fight against HIV/AIDS. First-line antiretroviral drugs (ARVs) have become more affordable, yet, assuring lifetime treatment requires the provision of expensive second and third-line therapy. Considering the longterm perspective, this analysis examines ARV mean cost evolution in Brazil and highlights main factors influencing its behaviour.

Methods Transactional data for ARV procurement made by the Brazilian Ministry of Health (BMoH) between 1998 and 2011 were used to calculate mean annual ARV cost, adjusted to 2011 US\$. 
Literature review on ARV price determinants and interviews with $\mathrm{BMoH}$ representatives have complemented the analysis.

Results From 1999 to 2003, thanks to local generic production and repeated compulsory licencing threats, total ARV expenditure fell by $56 \%$, despite patient increase of $72 \%$, which led to a $74 \%$ decrease in the cost per patient per year. Following the inclusion of third-line ARVs, in 2005, total expenditure peaked at about US\$ 460 million (2011 US\$) for the treatment of nearly 160,000 patients. Although the compulsory licencing of Efavirenz, in 2007, together with assertive price negotiations on patented drugs led to a significant drop in costs, most recently, as the number of patients continues to increase, local production remains highly uncompetitive, and, new-generation patented drugs are further being incorporated, mean annual cost has recovered speed, reaching values 35\% higher than 2003.

Conclusion The downward trend in mean ARV costs reached an inflexion point in 2003, after when limited savings from generic production and originator firm discounts were no longer able to compensate for the incorporation of highly expensive second and third-line ARVs. This finding reinforces patent protection being a major barrier to quality and longterm treatment in developing countries. In the case of Brazil, it further calls for higher efficiency on local production capacity.

\subsection{A SUCCESSFUL MODEL IN REACHING OUT MOBILE POPULATION TO CONTROL THE SPREAD OF STI/HIV/AIDS: EXPERIENCE FROM LINK WORKER SCHEME IMPLEMENTED IN 200 VILLAGES MOSTLY INHIBITED BY MOBILE POPULATION IN WEST BENGAL, INDIA}

doi:10.1136/sextrans-2013-051184.0133

'B Panda, ${ }^{2} \mathrm{D}$ Ghosh, ${ }^{3} \mathrm{~A}$ Ishlam, ${ }^{4} \mathrm{R}$ Kundu, ${ }^{5} \mathrm{M}$ Mahato. ${ }^{1}$ Chandipur Mother \& Child Welfare Society, Kolkata, India; ${ }^{2}$ Sristy for Human Society, Kolkata, India; ${ }^{3}$ Murshidabad District HIV Prevention Cell, Murshidabad, India; ' $P$ Purulia Zilla Parisad, Purulia, India; ${ }^{5}$ Gandhi Memorial Leprosy Foundation, Kolkata, India

The fact is that over $57 \%$ of the 2.9 million HIV positive people in India live in rural areas. India still has a rural base with $69 \%$ of its population living in rural areas. Young migrant workers and other highly mobile population essentially come from villages. Due to rural-urban continuum, rural population is also not lagging behind its urban counterpart in adopting high-risk behaviours.

To face the challenge of HIV/AIDS in rural India is even more difficult due to poor literacy rates resulting in poor awareness, mostly engaged in unorganised sector and stigma associated with it.

Link Workers Scheme (LWS) implemented in 100 villages each at Purulia \& Murshidabad targeting youth, vulnerable and high risk population for prevention of STI/HIV/AIDS and improve service delivery linkages. Murshidabad \& Purulia is a vulnerable district due to high migration, interstate border, trafficking, poverty, illiteracy and poor health care. Link Workers have been accepted as frontline development functionaries and are proving to be successful in reaching out to the target population.

LWS successfully reached out 4861 HRGs and 146079 vulnerable young men and women in last two years. Referral services to ICTC, STI and TB have increased significantly and a total of about 18500 cases referred to health facilities for testing of HIV, 79\% cases actually turned up for testing; more than 7800 STI cases identified, $64 \%$ reported; 2700 TB cases identified, $52 \%$ reported and utilised the services. About 3860 pregnant women identified in the project area and referred for HIV testing.

The link-worker, through a due diligence process, keeps on motivating the person to access HIV/AIDS related services such as HIV testing, treatment of STIs, ART, treatment of opportunistic infections and minimise the gap between beneficiary and service delivery. This need to scalled up to arrest the spread of STI/HIV/AIDS in rural setting.
009.4 MOBILIZATION FOR HIV, SYPHILIS AND HEPATITIS B AND C TESTING DURING BRAZIL'S CARNIVAL

doi:10.1136/sextrans-2013-051184.0134

A Bolzan, E Santos, D Serafim, E Ayer, A Neto, M Chaves, V Araújo, J Cerqueira. Ministry of Health, Brasilia, Brazil

Introduction Testing campaigns (entitled "Get to know") during high-traffic public events have been conducted in Brazil since July 2008. On these occasions, people are offered information about health and prevention and rapid tests (RTs), as well as condoms and lubricating gels. Initially, only rapid HIV testing was conducted; RTs for syphilis screening was included in 2010 and hepatitis B and C screening in 2012. Twenty-two national mobilizations were organised between 2008 and 2012, with approximately 26,300 RTs performed.

Methodology A large number of people attend the carnival celebrations in the cities of Recife and Salvador and are the focus of Ministry of Health campaigns. In 2013, for the first time, the "Get to Know" campaign in these cities offered RTs for HIV, syphilis, and hepatitis B and C. Testing was conducted in health centres near places where large numbers of people congregated and at temporary centres at the carnival venues. In cases with RT reactive results, patients received the test results and were referred to qualified services for follow-up and healthcare.

Results More than 8,000 tests were performed during the four days of carnival in Recife and Salvador. In Salvador, 5,280 tests were conducted in three locations, and 1,458,984 condoms were distributed by eleven mobile teams. Of those tested, 16 were reactive for HIV, 84 for syphilis, 7 for hepatitis B, and 14 for hepatitis C. In Recife, 5,527 RTs were performed in 3 locations, and 800,000 condoms were distributed. Twenty people were reactive for HIV, 60 for syphilis, 2 for hepatitis B, and 4 for hepatitis C.

Conclusion The "Get to Know" campaign has been proven efficient in scaling up access to early diagnosis and reaching large numbers of people with information about the importance of getting tested and with awareness-raising activities about prevention.

\subsection{OPTIMIZING CARE EFFICIENCY AT THE STI CLINIC: USING CHLAMYDIA HOME COLLECTION KITS}

doi:10.1136/sextrans-2013-051184.0135

R Koekenbier, A Hendriks, M van Veen, P van Leeuwen. Public Health Service Amsterdam, Amsterdam, The Netherlands

Background To study the efficiency of Chlamydia home collection kits for young people, in order to optimise care at the Amsterdam STI clinic.

Methods Since 2012, young people under the age of 25 with a low risk profile for STIs are only tested for Chlamydia. Tests for other STIs are indicated after testing Chlamydia positive. From September-November 2012, young people using the online intake were offered two different ways of Chlamydia testing: receiving a home collection kit, or coming to the clinic. The collection kit is send to the client by mail and is used to collect a swab or urine sample. This sample is send back to the laboratory for testing. The client can retrieve the results online by using the provided login.

Results In the study period, 523 online requests were done. Of these, 388 (74\%) opted for the home collection kit and $135(26 \%)$ preferred an appointment at the clinic. Of the requested kits, $86 \%$ were send back. All clients checked their test result online. Chlamydia was diagnosed in $5.5 \%$ of the clients receiving a home kit and in $2.9 \%$ of those tested at the clinic. Women were more likely to request a home collection kit $(77 \%$ versus $60 \%$ of men, $\mathrm{p}<0.001)$ as were young people aged $20-24$ years $(76 \%$ versus $64 \%$ aged $<20$ 